\title{
Bandwidth, intensity, and lineshape of the transmission spectrum in the single molecular junction
}

\author{
Liang-Yan Hsu, Bih-Yaw Jin * \\ Department of Chemistry and Center of Theoretical Sciences, National Taiwan University, Taipei 10617, Taiwan
}

\section{A R T I C L E I N F O}

\section{Article history:}

Received 26 February 2008

In final form 7 April 2008

Available online 11 April 2008

\begin{abstract}
A B S T R A C T
Using first order perturbation theory, we show that the bandwidth, intensity, and lineshape in transmission spectra of a single molecular junction calculated by the non-equilibrium Green's function (NEGF) method can be rationalized by the one- and two-resonant state approximations. Particularly, we find that the lineshape of the transmission function is strongly influenced by the relative phases between the two nearestneighbor resonant states. Finally, we utilize these results to examine two systems: a Hückel model system with five sites and a single fluoro-1,4-benzenedithiol molecule within the extended Hückel approximation. (C) 2008 Published by Elsevier B.V.
\end{abstract}

\section{Introduction}

With the advances of self-assembling techniques and scanning tunneling microscopes, the molecular electronics has given rise to extensive investigations [1-7] owing to its potential applications to nanoelectronic devices [4,8-10]. Among many different molecular electronic devices, the single molecular junction is the simplest and is composed by two electrodes and a molecular wire. Over the last decade, it has been found that transport properties of the single molecular junction are strongly influenced by the types of contacts $[11,12]$ and the electronic structure of the bridged molecule $[13,14]$. Therefore, it is important to understand the correlation between the transport properties and molecular electronic structures because it may provide us a guide to design appropriate molecular electronic devices.

In this Letter, we will start with the Landauer formula, and consider only the elastic electron scattering between the molecule and two electrodes in the weak coupling limit. From the Landauer formula and within the NEGF formalism, the transmission function can be represented as the follows

$T(E)=\operatorname{Tr}\left(\Gamma_{\mathrm{L}} G^{\mathrm{R}} \Gamma_{\mathrm{R}} G^{\mathrm{A}}\right)$,

where $G^{\mathrm{R}(\mathrm{A})}$ is the retarded (advanced) Green's function and $\Gamma_{\mathrm{L}(\mathrm{R})}$ is the spectral function of the left (right) electrode. The spectral function is related to the imaginary part of the self-energy, $\Gamma_{\mathrm{L}(\mathrm{R})}=$ $\mathrm{i}\left(\Sigma_{\mathrm{L}(\mathrm{R})}^{\mathrm{R}}-\Sigma_{\mathrm{L}(\mathrm{R})}^{\mathrm{A}}\right)=2 \Delta_{\mathrm{L}(\mathrm{R})}$, which can be calculated by the NewnsAnderson Model [15] or the surface Green's function [16,17]. The retarded Green's function is

$G^{\mathrm{R}}=\frac{1}{E-H_{\mathrm{mol}}-\Sigma^{\mathrm{R}}}$,

\footnotetext{
* Corresponding author.

E-mail address: byjin@ntu.edu.tw (B.-Y. Jin).
}

where $H_{\text {mol }}$ is the molecular Hamiltonian and $\Sigma^{\mathrm{R}}$ is the retarded self-energy contributed from two electrodes. In the weak coupling limit and using the tight-binding approximation, the Green's function in Eq. (2) can be approximated by first order perturbation theory [18] as

$$
\begin{aligned}
& \left(G^{\mathrm{R}}\right)_{\mu v}=\sum_{n} \frac{C_{\mu n}^{(0) *} C_{v n}^{(0)}}{E-\varepsilon_{n}^{(0)}-\varepsilon_{n}^{(1)}}, \\
& \varepsilon_{n}^{(1)}=\left\langle n^{(0)}\left|\Sigma^{\mathrm{R}}\right| n^{(0)}\right\rangle=\sum_{\mu v} C_{\mu n}^{(0)} C_{v n}^{(0)} \Sigma_{\mu v}^{\mathrm{R}},
\end{aligned}
$$

where we have written the molecular orbital, $\left|n^{(0)}\right\rangle$, as a linear combination of atomic orbitals, $\left|n^{(0)}\right\rangle=\sum_{\mu} C_{\mu n}^{(0)}|\mu\rangle$. Eqs. (1) and (3) are the starting point in the next two sections.

\section{Bandwidth and intensity: one-resonant-state approximation}

In order to obtain the analytical forms for the bandwidth and the intensity in the transmission spectrum, we consider only one orbital on each site, nearest-neighbor couplings and neglect of the electron correlation, hence the total Hamiltonian is written as

$H_{\text {total }}=H_{\text {mol }}+H_{\text {electrode }}+H_{\text {coupling }}$,

$H_{\mathrm{mol}}=\sum_{\mu v}^{N} \varepsilon_{\mu v} a_{\mu}^{\dagger} a_{v}$,

$H_{\text {electrode }}=\sum_{i} \varepsilon_{i} a_{i}^{\dagger} a_{i}+\sum_{j} \varepsilon_{j} a_{j}^{\dagger} a_{j}$,

$H_{\text {coupling }}=\sum_{i} \beta_{i}\left(a_{1}^{\dagger} a_{i}+c . c\right)+\sum_{j} \beta_{j}\left(a_{N}^{\dagger} a_{j}+\right.$ c.c $)$,

where 1 and $\mathrm{N}$ stand for the junction atom orbitals; $i$ and $j$ stand for the left and the right electrode orbitals. According to the nearestneighbor coupling approximation, Eq. (1) turns into 


$$
\begin{aligned}
T(E) & =\left(\Gamma_{\mathrm{L}}\right)_{11}\left(G^{\mathrm{R}}\right)_{1 N}\left(\Gamma_{\mathrm{R}}\right)_{N N}\left(G^{\mathrm{A}}\right)_{N 1} \\
& =4\left(\Delta_{\mathrm{L}}\right)_{11}\left(\Delta_{\mathrm{R}}\right)_{N N}\left(G^{\mathrm{R}}\right)_{1 N}\left(G^{\mathrm{A}}\right)_{N 1},
\end{aligned}
$$

and the retarded Green's function becomes

$$
\left(G^{\mathrm{R}}\right)_{1 N}=\sum_{n} \frac{C_{1 n}^{(0) *} C_{N n}^{(0)}}{E-\varepsilon_{n}^{(0)}-\left|C_{1 n}^{(0)}\right|^{2}\left(\Lambda_{11}-\mathrm{i} \Delta_{11}\right)-\left|C_{N n}^{(0)}\right|^{2}\left(\Lambda_{N N}-\mathrm{i} \Delta_{N N}\right)},
$$

where $\Lambda$ is the real part of the self-energy. Suppose the energy of an incident electron is close to a certain resonant state $a$, and is far away from the other resonant states. It is reasonable to neglect the contribution from the other resonant states, and Eq. (6) can be approximated as

$$
\left(G^{\mathrm{R}}\right)_{1 N} \approx \frac{C_{1 a}^{(0) *} C_{N a}^{(0)}}{E-\varepsilon_{a}^{(0)}-\left|C_{1 a}^{(0)}\right|^{2}\left(\Lambda_{11}-\mathrm{i} \Delta_{11}\right)-\left|C_{N a}^{(0)}\right|^{2}\left(\Lambda_{N N}-\mathrm{i} \Delta_{N N}\right)},
$$

and the transmission function can be calculated as

$T(E)$

$$
=\frac{4 \Delta_{11} \Delta_{N N}\left|C_{1 a}^{(0)}\right|^{2}\left|C_{N a}^{(0)}\right|^{2}}{\left(E-\varepsilon_{a}^{(0)}-\left(\left|C_{1 a}^{(0)}\right|^{2} \Lambda_{11}+\left|C_{N a}^{(0)}\right|^{2} \Lambda_{N N}\right)\right)^{2}+\left(\left|C_{1 a}^{(0)}\right|^{2} \Delta_{11}+\left|C_{N a}^{(0)}\right|^{2} \Delta_{N N}\right)^{2}} .
$$

It is evident that the intensity of the transmission function is related to the MO coefficients, spectral density, and the position of the resonant state energy. For simplicity, if we neglect the energy shift term and assume that two electrodes and the electrode-molecular contacts are exactly the same, and also ignore the energy dependence of the self-energy, thus we have $\Delta_{11}=\Delta_{N N}=\alpha$, where $\alpha$ is a constant. Eq. (9) becomes

$$
T(E) \approx \frac{4 \alpha^{2}\left|C_{1 a}^{(0)}\right|^{2}\left|C_{N a}^{(0)}\right|^{2}}{\left(E-\varepsilon_{a}^{(0)}\right)^{2}+\alpha^{2}\left(\left|C_{1 a}^{(0)}\right|^{2}+\left|C_{N a}^{(0)}\right|^{2}\right)^{2}} \leqslant 1 .
$$

It is obvious that the inequality in Eq. (9) is ensured by the CauchySchwarz inequality, $4\left|C_{1 a}^{(0)}\right|\left|C_{\mathrm{Na}}^{(0)}\right| \leqslant\left|C_{1 a}^{(0)}\right|^{2}+\left|C_{\mathrm{Na}}^{(0)}\right|^{2}$. The maximum of transmission functions occurs only when $C_{1 a}^{(0)}=C_{N n}^{(0)}$ and the incident electron is exactly on resonance, i.e. $E-\varepsilon_{a}^{(0)}$. In other words, the total transmission can never occur in the non-symmetric resonant states $\left(C_{1 a}^{(0)} \neq C_{\mathrm{Na}}^{(0)}\right)$. Additionally, this formula can also explain that the
Eq. (9) can be extended to the situation that each site has more than one orbital. Suppose the off-diagonal terms of $\Sigma_{\mathrm{L}(\mathrm{R})}$ are important, the transmission functions can then be generalized as follows

$$
T(E) \approx \frac{4 \alpha^{2} \sum_{\mu v}\left|C_{\mu n}^{(0)}\right|^{2}\left|C_{v n}^{(0)}\right|^{2}}{\left(E-\varepsilon_{n}^{(0)}\right)^{2}+\alpha^{2}\left(\sum_{\mu}\left|C_{\mu n}^{(0)}\right|^{2}+\sum_{v}\left|C_{v n}^{(0)}\right|^{2}\right)^{2}}
$$

Notice that the off-diagonal terms of $\Sigma_{\mathrm{L}(\mathrm{R})}$ can have a nontrivial influence on the transmission function in some special cases [18]. Nevertheless, Eq. (10) still provides us a preliminary analysis to determine which MO is a major resonant state in the single molecular junction.

\section{Lineshape: two-resonant-state approximation}

The one-resonant-state approximation can explain the bandwidth and the intensity of the transmission function. In order to further understand the origin of the lineshape of the transmission function, we have to go beyond the one-resonant-state approximation, and consider at least two resonant states in Eq. (6), a and b, which are close to the energy of the incident electron. Similarly, by ignoring the term of energy shift and the energy dependence of the self-energy, $\Delta_{11}=\Delta_{N N}=\alpha$, Eq. (6) becomes

$$
\left(G^{\mathrm{R}}\right)_{1 N} \approx \sum_{n=a, b} \frac{C_{1 n}^{(0) *} C_{N n}^{(0)}}{E-\varepsilon_{n}^{(0)}+\mathrm{i} \alpha\left(\left|C_{1 n}^{(0)}\right|^{2}+\left|C_{N n}^{(0)}\right|^{2}\right)} .
$$

Substituting Eq. (11) into Eq. (5), we found that the transmission function can be divided into a symmetric and an asymmetric parts:

$T(E)=T_{\text {sym }}(E)+T_{\text {asym }}(E)$.

The symmetric part of the transmission function can be regarded as the superposition of two Lorentzian-type peaks:

$$
T_{\text {sym }}(E)=\sum_{n=a, b} \frac{4 \alpha^{2}\left|C_{1 n}^{(0)}\right|^{2}\left|C_{N n}^{(0)}\right|^{2}}{\left(E-\varepsilon_{n}^{(0)}\right)^{2}+\alpha^{2}\left(\left|C_{1 n}^{(0)}\right|^{2}+\left|C_{N n}^{(0)}\right|^{2}\right)^{2}} .
$$

When the energy of incident electron is close to one of the resonant states, the symmetric part of the transmission function will reduce to Eq. (9). However, in order to obtain the asymmetric part of the transmission function, we have to take advantage of the fact that the MO coefficients, $C_{\mu \nu}^{(0)}$, are real. Therefore, we have

$$
\begin{aligned}
& T_{\text {asym }}(E)=\frac{4 C_{1 a}^{(0)} C_{N a}^{(0)} C_{1 b}^{(0)} C_{N b}^{(0)}\left[\left(E-\varepsilon_{a}\right)\left(E-\varepsilon_{b}\right)+\alpha^{2} C_{1 a}^{(0)} C_{N a}^{(0)} C_{1 b}^{(0)} C_{N b}^{(0)}\right]}{\left[\left(E-\varepsilon_{a}^{(0)}\right)^{2}+\alpha^{2}\left(\left|C_{1 a}^{(0)}\right|^{2}+\left|C_{N a}^{(0)}\right|^{2}\right)^{2}\right]\left[\left(E-\varepsilon_{b}^{(0)}\right)^{2}+\alpha^{2}\left(\left|C_{1 b}^{(0)}\right|^{2}+\left|C_{N b}^{(0)}\right|^{2}\right)^{2}\right]} \\
& \approx \frac{4 C_{1 a}^{(0)} C_{N a}^{(0)} C_{1 b}^{(0)} C_{N b}^{(0)}\left(E-\varepsilon_{a}\right)\left(E-\varepsilon_{b}\right)}{\left[\left(E-\varepsilon_{a}^{(0)}\right)^{2}+\alpha^{2}\left(\left|C_{1 a}^{(0)}\right|^{2}+\left|C_{N a}^{(0)}\right|^{2}\right)^{2}\right]\left[\left(E-\varepsilon_{b}^{(0)}\right)^{2}+\alpha^{2}\left(\left|C_{1 b}^{(0)}\right|^{2}+\left|C_{N b}^{(0)}\right|^{2}\right)^{2}\right]} .
\end{aligned}
$$

molecular orbitals with the MO coefficients satisfying $C_{1 a}^{(0)} \gg C_{N a}^{(0)}$ or $C_{1 a}^{(0)} \ll C_{\mathrm{Na}}^{(0)}$ make little contributions to electric conduction. Moreover, we can use Eq. (9) to obtain the approximate half-width of peaks of various resonant states without complicated calculations. It is noteworthy that when the energy of an incident electron is close to a symmetric resonant state $\left(C_{1 a}^{(0)}=C_{\mathrm{Na}}^{(0)}\right)$, the half-width of each peak can be simplified as $2 \alpha C_{1 a}^{(0) 2}$.
In simplifying Eq. (15) from Eq. (14), we have assumed that the second term of the numerator in Eq. (14) is much smaller than the first term in the weak coupling condition, $\alpha \ll 1$ and far away from these two resonances, and can thus be neglected. Additionally, since the denominator in Eq. (14) is positive, thus, the value of the asymmetric term is determined mainly by the MO coefficients of the two nearest-neighbor resonant states and the energy of the 


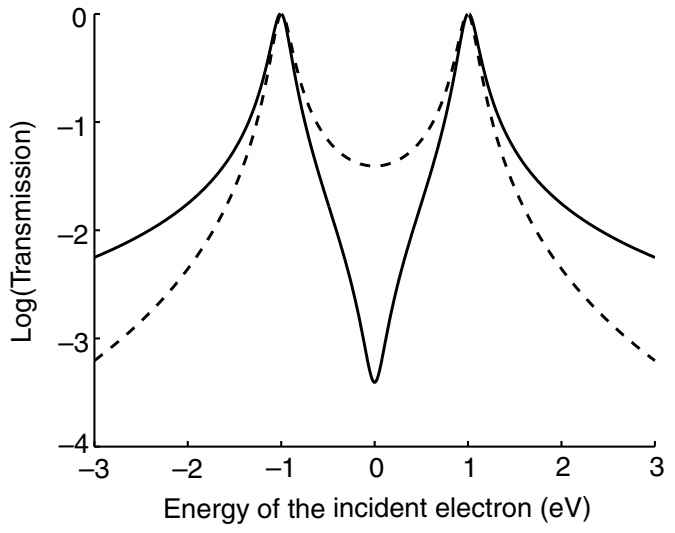

Fig. 1. The total transmission function of the two resonant state model. The parameters: $\alpha=1, \varepsilon_{a}=-1, \varepsilon_{b}=1, C_{1 a}^{(0)}=1 / \sqrt{2}, C_{N a}^{(0)}=1 / \sqrt{2}$, and $C_{1 b}^{(0)}=1 / \sqrt{2}$. The solid line: $C_{b N}^{(0)}=1 / \sqrt{2}$. The dashed line: $C_{N b}^{(0)}=-1 / \sqrt{2}$.

incident electron. The total transmission function of the two-resonant-state model is shown in Fig. 1.

Let us consider the situation when the energy of an incident electron is located in between these two resonances, $\varepsilon_{a}<\varepsilon_{b}$, as shown in Fig. 1. If the phases of the two resonant states satisfy $C_{1 a}^{(0)} C_{N a}^{(0)} C_{1 b}^{(0)} C_{N b}^{(0)}>0$, the magnitude of transmission function in this region is strongly suppressed due to destructive interference. On the contrary, if $C_{1 a}^{(0)} C_{N a}^{(0)} C_{1 b}^{(0)} C_{N b}^{(0)}<0$, the magnitude of transmission function in this region is enhanced due to constructive interference.

\section{A simple model system}

To illustrate how our previous results can be employed, here we consider a simple one-dimensional molecular wire that contains five sites with one orbital on each site. In the tight-binding approximation, the molecular Hamiltonian can be written as

$H_{\mathrm{mol}}=\epsilon \sum_{i=1}^{5} a_{i}^{\dagger} a_{i}-t \sum_{i=1}^{4}\left(a_{i}^{\dagger} a_{i+1}+\right.$ h.c $)$.

A
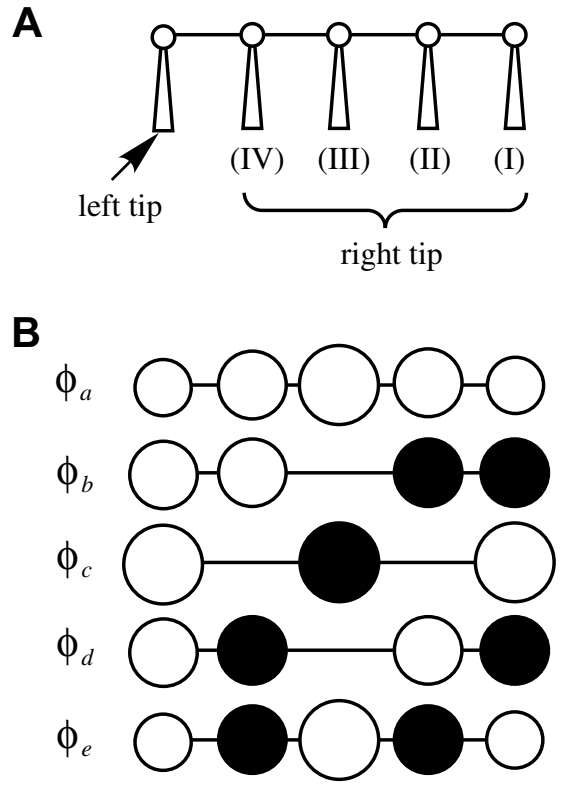

Fig. 2. (A) The molecular wire connected with two tips. (B) Five molecular orbitals of the corresponding molecular wire.
Now let's consider a gedanken experiment as shown in Fig. 2A, in which the molecular wire is connected by two tips. Assume that the left tip is fixed and the right tip can be moved to four different positions, (I), (II), (III), and (IV). Fig. 2B shows the five molecular orbitals of the molecular wire in increasing energy. The black and white colors stand for the positive and negative phases of the orbitals. The size of a circle represents the relative magnitude of the corresponding atomic orbital.

The calculation of the transmission spectra is based on the NEGF method. For simplicity, the real part of the self-energy is assumed to be zero and the imaginary part a constant. The calculated transmission spectra for these four hypothetical situations are shown in Fig. 3. These figures clearly exhibit that the different positions of the right tip can affect the shapes of transmission spectra enormously. Here we will demonstrate that these non-trivial changes can be interpreted by the one- and two-resonant-state approximation introduced in the previous sections.

The gross features of Fig. $3 \mathrm{~A}-\mathrm{C}$ such as the positions, intensities, and bandwidths of transmission resonances can be well understood by the one-resonant-state approximation. For instance, the five resonances in Fig. $3 \mathrm{~A}$ occur exactly at the eigenvalues of the molecular Hamiltonian due to the neglect of the real part of the self-energy. The relative magnitudes of bandwidth for each transmission resonance are roughly in proportion to the $\left|C_{1 a}^{(0)}\right|^{2}+$ $\left|C_{\mathrm{Na}}^{(0)}\right|^{2}$, which is consistent with the prediction based on Eq. (9). Unlike the hypothetical experiment (I), the transmission spectra for (II) and (III) as shown in Fig. 3B and C exhibit interesting antiresonances at the molecular orbitals with vanishing amplitude right at the position of the right tip. The transmission suddenly drops to zero when the energy of the incident electron is close to the antiresonance. Based on the molecular orbitals as shown Fig. 2, there is no antiresonance for experiment (I), exactly one antiresonance at $0 \mathrm{eV}$ for experiment (II) and (IV), and two antiresonances at $\pm 1 \mathrm{eV}$ for experiment (III).

Finally, we can see that the transmission spectrum for the experiment (IV) shows additional change in its lineshape between the transmission resonances, $-1.732 \mathrm{eV}$ and $-1 \mathrm{eV}$. The origin of this mis-shape can be understood only through the two-resonant-state approximation. In this case, the right tip is put right next to the left tip. We can easily see that the intensities of the peaks at $-1 \mathrm{eV}$ and $1 \mathrm{eV}$ reach the maximum since the MO coefficients on the first and the second atoms are equal (see $\phi_{b}$ and $\phi_{d}$ in Fig. 2B). The shape between the transmission resonances, $-1.732 \mathrm{eV}$ and $-1 \mathrm{eV}$, is strongly distorted owing to the MO coefficients conforming to the requirement of destructive interference, $C_{1 a}^{(0)} C_{N a}^{(0)} C_{1 b}^{(0)} C_{N b}^{(0)}>0$ (see $\phi_{a}$ and $\phi_{b}$ in Fig. 2B). In addition, the fact that the width is the peak at $-1 \mathrm{eV}$ is larger than that of the peak at $-1.732 \mathrm{eV}$ can be explained due the the larger magnitude of the MO coefficients.

From the calculation based on this simple model, we have found that the single molecule conductance is strongly influenced by the position of the connection, in addition to the molecular structure, the electrodes and the junction atoms. This kind of sensitive dependence on the connection has been demonstrated in the recent experiments $[19,20]$.

\section{A single fluoro-1,4-benzenedithiol molecule}

In this section, we apply our rules to examine the transmission function of a single fluoro-1,4-benzenedithiol molecule (see Fig. 4). The transmission function is calculated by the NEGF method within the extended Hückel theory(the NEGF-EHT). The NEGF-EHT codes are based on the combination of the HuckelIV 2.0 [21] and the Yaehmop [22]. The sulfur atom of the molecular wire is assumed to be connnected to the hollow position of the 111 surface of Au electrodes, and the distance between the sulfur atom and $\mathrm{Au}$ 

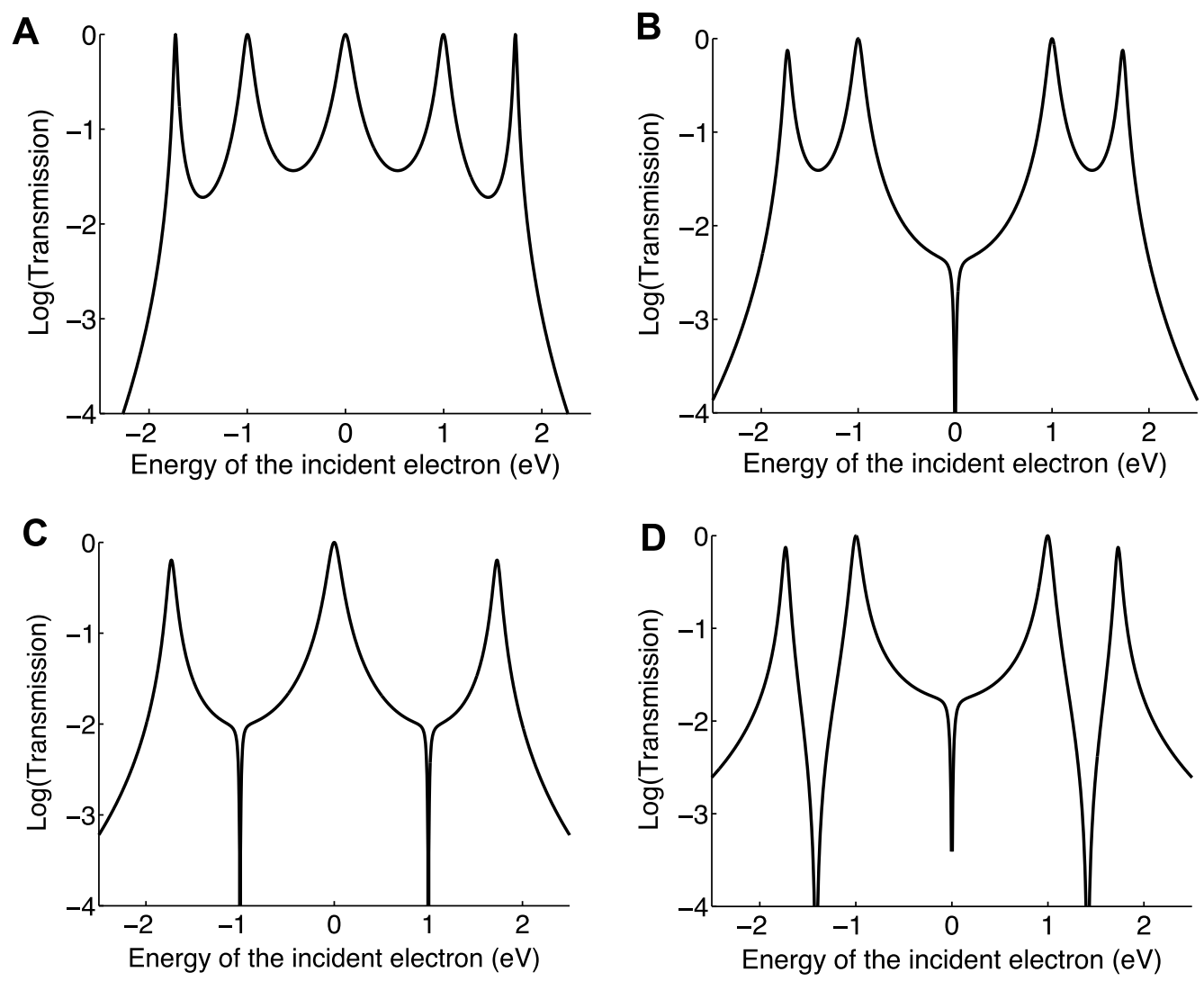

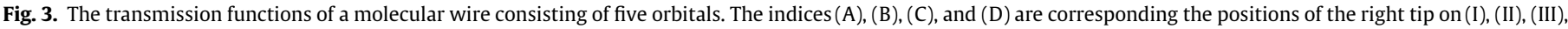
and (IV) in Fig. 2(B). Parameters: $\epsilon=0, t=1$, and $\Delta=0.1$.

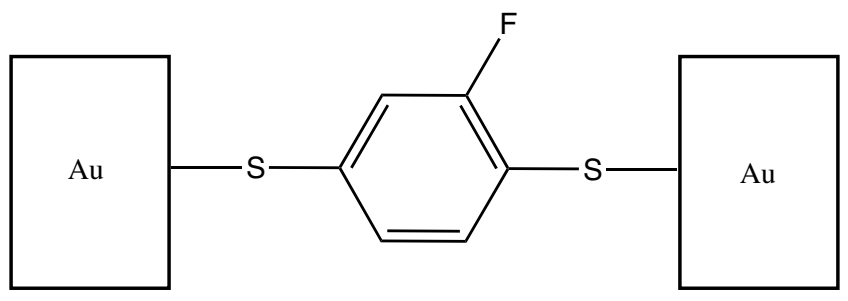

Fig. 4. Au-fluoro-1,4-benzenedithiol-Au junction.

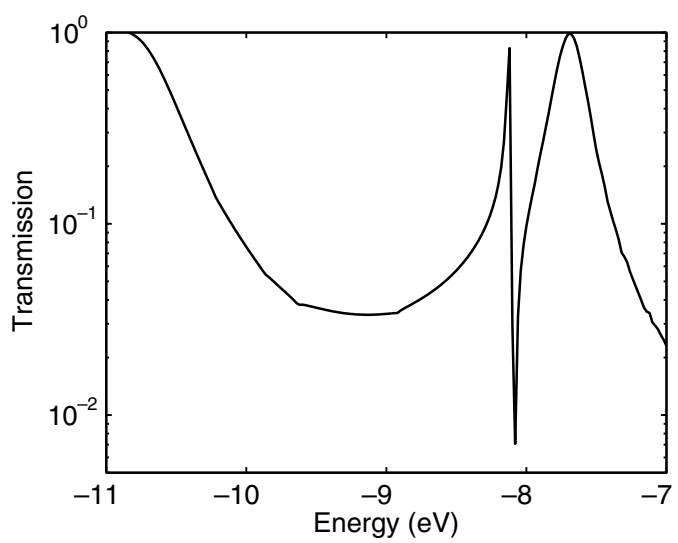

Fig. 5. The transmission function of a single fluoro-1,4-benzenedithiol molecule.
Table 1

The energy and MO coefficients of a fluoro-1,4-benzenedithiol molecule

\begin{tabular}{|c|c|c|c|c|c|c|}
\hline \multirow{2}{*}{$\frac{\text { Molecular Orbital }}{\text { Energy }}$} & \multicolumn{2}{|l|}{ A } & \multicolumn{2}{|l|}{ B } & \multicolumn{2}{|l|}{ C } \\
\hline & \multicolumn{2}{|c|}{-10.42} & \multicolumn{2}{|l|}{-8.11} & \multicolumn{2}{|l|}{-7.48} \\
\hline Types of Orbital & left & right & left & right & left & right \\
\hline s & 0.000 & 0.000 & 0.000 & 0.000 & 0.000 & 0.000 \\
\hline $\mathrm{p}_{\mathrm{x}}$ & 0.000 & 0.000 & 0.000 & 0.000 & 0.000 & 0.000 \\
\hline $\mathrm{p}_{\mathrm{y}}$ & 0.000 & 0.000 & 0.000 & 0.000 & 0.000 & 0.000 \\
\hline $\mathrm{p}_{\mathrm{z}}$ & 0.626 & -0.669 & 0.047 & 0.089 & -0.323 & -0.299 \\
\hline
\end{tabular}

Molecular orbital energy in eV calculated by Yaehmop.

The MO coefficients of the left sulfur atoms attached onto the left and right electrodes.

surface is chosen to be $1.905 \AA$, which is determined by the DFT optimization in previous work [23].

The calculated transmission spectrum is shown in Fig. 5. The two peaks located at $-7.6 \mathrm{eV}$ and $-8.2 \mathrm{eV}$, as shown in Fig. 5, correspond to the transmission through MOs of the fluoro-1,4-benzenedithiol with eigenvalues, $-7.4 \mathrm{eV}$ and $-8.1 \mathrm{eV}$, respectively. The peak located at $-10.8 \mathrm{eV}$ is related to the MO with energy, $-10.4 \mathrm{eV}$. In Table 1 , we list the energies and the corresponding MO coefficients on the sulfur atoms of these three MOs. It is easy to see from Table 1 that the atomic coefficients of these three MOs are all concentrated on the $\mathrm{p}_{z}$ orbital. Thus, it is adequate to apply the previous lineshape analysis to explain the transmission spectra of the conjugated systems.

The AO coefficients of the left and the right sulfur atoms for molecular orbitals, $\mathbf{A}$ and $\mathbf{C}$, are close to each other in magnitudes. Thus, the intensities of these two peaks in the transmission function are larger than the one due to the molecular orbital B. Additionally, the magnitudes of $\mathrm{MO}$ coefficients on $\mathbf{A}$ and $\mathbf{C}$ are greater than that on $\mathbf{B}$, so their bandwidths of the transmission function are wider 
than that of $\mathbf{B}$. Note also that the MO coefficients of $\mathbf{B}$ and $\mathbf{C}$ satisfy the condition of destructive interference, $C_{1 b}^{(0)} C_{N b}^{(0)} C_{1 c}^{(0)} C_{N c}^{(0)}>0$, so that the lineshape is strongly mis-shaped when close to resonance. On the other hand, the MO coefficients of $\mathbf{A}$ and $\mathbf{B}$ satisfy the condition, $C_{1 a}^{(0)} C_{N a}^{(0)} C_{1 b}^{(0)} C_{N b}^{(0)}<0$, thus, the lineshape decreases smoothly. These results are consistent with our theory.

In conclusion, our theory has not only successfully explained the characteristics of the transmission spectra, but also provided us a simple analysis of the intensity, linewidth, and lineshape in the transmission spectra based on a simple molecular orbital computation. However, owing to the neglect of electron correlations and vibronic couplings, our theory can predict neither the many body effects such as the Kondo effects and Coulomb blockade [4,6] nor the vibronic effects like inelastic scattering [24-27]. Albeit with the restriction of the independent electron approximation, we believe the theory we propose is still very useful for the understanding the transmission function. The deviation from this picture of the transmission spectrum indicates the importance of electron-electron correlation and the electron-phonon interaction.

\section{Acknowledgement}

We wish to thank the National Science Council, Taiwan for the financial support.

\section{References}

[1] M.A. Read, C. Zhou, C.J. Muller, T.P. Burgin, J.M. Tour, Science 278 (1997) 252.

[2] H. Ohnishi, Y. Kondo, K. Takayanagi, Nature 395 (1998) 780.
[3] A.I. Yanson, G.R. Bollinger, H. den Brom, N. Agrait, J. Ruitenbeek, Nature 395 (1998) 783.

[4] J. Park et al. , Nature 417 (2002) 722.

[5] R.H.M. Smit, Y. Noat, C. Untiedt, N.D. Lang, M. van Hemert, J. Ruitenbeek, Nature 419 (2002) 906.

[6] W.J. Liang, M.P. Shores, M. Bockrath, J.R. Long, H. Park, Nature 417 (2002) 6890.

[7] A. Nitzan, M.A. Ratner, Science 300 (2003) 1384

[8] A. Aviram, M.A. Ratner, Chem. Phys. Lett. 29 (1953) 277.

[9] C. Joachim, J.K. Gimzewski, A. Aviram, Nature 408 (2000) 541.

[10] N.J. Tao, Nature Nanotechnol. 1 (2006) 173.

[11] S.N. Yaliraki, A.E. Roitberg, C. Gonzalez, V. Mujica, M.A. Ratner, J. Chem. Phys. 111 (1999) 6997.

[12] G. Heimel, L. Romaner, E. Zojer, J.L. Bredas, Nano lett. 7 (2007) 932

[13] L. Venkataraman, M.S. Hybersten, J.E. Klare, C. Nuckolls, M.L. Steigerwald, Nature 442 (2006) 904

[14] L. Venkataraman, Y.S. Park, A.C. Whalley, C. Nuckolls, M.S. Hybertsen, M.L. Steigerwald, Nano lett. 7 (2007).

[15] V. Mujica, M. Kemp, M.A. Ratner, J. Chem. Phys. 101 (1994) 6856.

[16] M.A. Reed, Molecular Nanoelectronics, American Scientific Publishers, 2003.

[17] H. Morkoc, Advanced Semiconductors and Organic Nano-techniques, Academic, 2003.

[18] T. Shimazaki, H. Maruyama, Y. Asai, K. Yamashita, J. Chem. Phys. 123 (2005) 164111.

[19] J. Repp, G. Meyer, S.M. Stojkovic, A. Gourdon, C. Joachim, Phys. Rev. Lett. 94 (2005) 026803.

[20] P. Liljeroth, J. Repp, G. Meyer, Phys. Rev. Lett. 317 (2007) 1203.

[21] F. Zahid, Huckel-IV 2.0 Source Code Download, <http://www.nanohub.org/ resources/> .

[22] G.A. Lamdrum, W.V. Glassey, bind (ver 3.0).

[23] H. Sellers, A. Ulman, Y. Shnidman, J.E. Eilers, J. Amer. Chem. Soc. 115 (1993) 9389.

[24] A. Troisi, M.A. Ratner, A. Nitzan, J. Chem. Phys. 118 (2003) 6072.

[25] J. Koch, F. Oppen, Phys. Rev. Lett. 94 (2005) 206804.

[26] M. Galperin, A. Nitzan, M.A. Ratner, J. Phys. Cond. Mat. 19 (2007) 103201.

[27] R.H.M. Smit, Y. Noat, C. Untiedt, N.D. Lang, M. Hemert, J. van Ruitenbeek, Nature 419 (2002) 906 